\title{
ENSAIOS
}

\section{APRENDIZAGEM DO TREINADOR ESPORTIVO COMO UM PROCESSO DE PARTICIPAÇÃO SOCIAL: UMA PERSPECTIVA À PESQUISA CIENTÍFICA}

\begin{abstract}
SPORTS COACHES' LEARNING AS A SOCIAL PARTICIPATION PROCESS: A PERSPECTIVE FOR SCIENTIFIC RESEARCH C
\end{abstract}

EL APRENDIZAJE DEL ENTRENADOR DEPORTIVO COMO UN PROCESO DE PARTICIPACIÓN SOCIAL: UNA PERSPECTIVA PARA LA INVESTIGACIÓN CIENTÍFICA CP

do) https://doi.org/10.22456/1982-8918.105388

(D) Vinicius Zeilmann Brasil ${ }^{*}<$ vzbrasil@hotmail.com>

(1) Valmor Ramos* <valmor.ramos@udesc.br>

(D) Ana Flávia Backes** <anafbackes@hotmail.com>

(D) Leonardo Ristow*<leonardoristow@live.com>

(D) Michel Milistetd ${ }^{* *}<$ michel_canhoto@hotmail.com>

(D) Juarez Vieira do Nascimento** <juarez.nascimento@ufsc.br>
*Universidade do Estado de Santa Catarina (UDESC). Florianópolis, SC, Brasil. ${ }^{* *}$ Universidade Federal de Santa Catarina. Florianópolis, SC, Brasil.
Resumo: O objetivo deste ensaio teórico foi apresentar a Teoria Social da Aprendizagem (TSA) e indicar como o quadro conceitual adjacente tem sido utilizado nas investigações sobre a aprendizagem e o desenvolvimento de treinadores esportivos. A TSA oferece um escopo analítico e instrumental amplo, com potencial para a compreensão e a promoção da aprendizagem do treinador. Os direcionamentos temáticos emergentes na agenda investigativa nesta área refletem a amplitude do quadro conceitual que constitui a TSA. As contribuições destes estudos contemplam a demanda de se utilizar matrizes teóricas que preservem a complexidade, a natureza social e o caráter contextual da aprendizagem dos treinadores.

Palavras chave: Aprendizagem. Educação Física e Treinamento. Esportes. Teoria Social.
Recebido em: 14 jul. 2020 Aprovado em: 30 abr. 2021 Publicado em: 09 jun. 2021

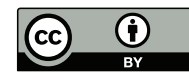

Este é um artigo publicado sob a licença Creative Commons Atribuição 4.0 Internacional (CC BY 4.0). eISSN: $1982-8918$ 


\section{INTRODUÇÃO}

O processo de aprendizagem profissional do treinador é reconhecido como um elemento central na busca pela qualidade da intervenção pedagógica no esporte (GILBERT; TRUDEL, 2004). As experiências como atleta e como treinador, as interações sociais e o mentoring informal são destacadamente úteis à aprendizagem do treinador (BRASIL et al., 2015; WALKER; THOMAS; DRISKA, 2018). Adicionalmente, as críticas à efetividade dos programas de formação de treinadores (MALLETT et al., 2009), o advento das tecnologias da informação e de comunicação (CUSHION; TOWNSEND, 2019) e a necessidade de compreender a influência das experiências dos treinadores na sua intervenção profissional (CALLARY; WERTHNER; TRUDEL, 2013) são demandas que evocam renovados patamares de investigação científica nesta área.

A valorização da aprendizagem informal tem impulsionado uma perspectiva que reconhece o caráter social e contextualizado da aprendizagem (ARMOUR, 2010), direcionando o foco das análises para o modo específico com que o treinador participou/participa e interagiu/interage com diferentes indivíduos em determinadas práticas e contextos sociais (TRUDEL; GILBERT, 2006). Tais constatações indicam a relevância de uma perspectiva de aprendizagem em que a relação entre treinador e os contextos sociais em que ele participa definem a construção dos seus conhecimentos para a intervenção pedagógica. Isto abrange um processo contínuo e sucessivo de engajamento em práticas sociais, de mudanças em termos de competências e habilidades úteis à profissão, de incorporação de valores (crenças), assim como o desenvolvimento de perspectivas pessoais sobre a vida e sobre sua própria atuação enquanto treinador (CUSHION; TOWNSEND, 2016; MALLETT, 2010).

Este entendimento revela uma orientação socioconstrutivista da aprendizagem do treinador (CASSIDY; ROSSI, 2006; MALLETT, 2010) e que tem sido interpretada por meio da Teoria Social da Aprendizagem (TSA) (Social Theory of Learning) e do conceito de Comunidade de Prática (CdP) (Communities of Practice), propostos por Wenger (1998). Uma CdP compreende um "espaço de aprendizagem social" simples, constituinte de uma "paisagem de prática" que inter-relaciona outros diversos tipos de espaços de aprendizagem social (networks, discussões e equipes de trabalho) (WENGER-TRAYNER; WENGER-TRAYNER, 2018).

Esses conceitos compõem um amplo quadro teórico em que a aprendizagem é concebida como um tipo de "viagem" do indivíduo em uma "paisagem social" constituída por várias práticas sociais interconectadas, resultando no desenvolvimento de: competências de participação nos diferentes contextos dessa paisagem; de uma trajetória de identidade; de interesses, valores, perspectivas pessoais e profissionais; e de projeções futuras de participação (WENGER, 2010). Desse modo, a aprendizagem se manifesta e se consolida na própria forma de participação do indivíduo nessa "paisagem social", abrangendo o seu nível de engajamento nas diferentes práticas sociais, o nível de competência alcançado em relação a essas práticas e na sua capacidade de transitar entre os diferentes contextos e práticas sociais (WENGER-TRAYNER et al., 2015). 
Estudos evidenciam que os sistemas esportivos são constituídos por diferentes CdP (CULVER; TRUDEL, 2008; GALIPEAU; TRUDEL, 2006) e que as competências e a identidade do treinador resultam da sua trajetória de participação em diferentes CdP ao longo da sua vida (BRASIL, 2019; BRASIL et al., 2018; DUARTE; CULVER; PAQUETE, 2020). As pesquisas com caráter instrumental revelam a efetividade do desenvolvimento de CdP entre treinadores de uma mesma equipe ou clube e a importância de um facilitador para "nutrir" o engajamento dos treinadores nas iniciativas da CdP (CALLARY, 2013; CULVER; TRUDEL, 2006; CULVER; TRUDEL; WERTHNER, 2009). Além disso, a promoção de interações sociais entre treinadores potencializa a aprendizagem por meio do processo de reflexão (STOSZKOWSKI; COLLINS, 2014; KUKLICK et al., 2016). Portanto, a ênfase na participação social confere à TSA um relevante potencial para a análise da aprendizagem do treinador (CULVER; KRAFT, 2017).

Assim, busca-se por meio deste ensaio teórico apresentar a Teoria Social da Aprendizagem (TSA) (WENGER, 1998) e indicar como o quadro conceitual adjacente tem sido utilizado nas investigações sobre a aprendizagem e o desenvolvimento de treinadores esportivos, contribuindo para a ampliação das possibilidades investigativas e do debate científico na área. Para tanto, serão apresentados: a) os pressupostos, os componentes conceituais e os processos adjacentes da TSA; b) as implicações da TSA nas pesquisas com foco na aprendizagem e no desenvolvimento de treinadores esportivos; e por fim, c) as perspectivas e os desafios para a pesquisa científica na área.

\section{TEORIA SOCIAL DA APRENDIZAGEM (TSA)}

A Teoria Social da Aprendizagem (TSA) (Social Theory of Learning) (WENGER, 1998) foi desenvolvida a partir da noção de apprenticeship ${ }^{1}$ e da formulação dos conceitos de Aprendizagem Situada (Situated Learning) e Comunidade de Prática (CdP) (Communities of Practice), propostos por Lave e Wenger (1991). Sob influência de uma perspectiva socioconstrutivista da aprendizagem ${ }^{2}$, Lave e Wenger (1991) elucidaram o processo relacional entre a pessoa e o mundo, propondo que, ao interagirem, os indivíduos movem-se de uma participação periférica a uma participação mais central nas práticas de uma $\mathrm{CdP}$, caracterizando o que eles denominaram de Participação Legítima Periférica (Legitimate Peripheral Participation). Na TSA, a noção de CdP consiste em um conceito central no qual, segundo Wenger (1998, Prefácio, tradução nossa), "[...] a unidade primária de análise não é nem o indivíduo e nem as instituições sociais, mas as comunidades de prática informais que as pessoas constituem".

\footnotetext{
1 Apprenticeship corresponde à antiga prática de aprender um ofício ou uma profissão por meio de treinamento (formação) no trabalho, sob a supervisão de um profissional ou mestre experiente (LAVE; WENGER, 1991). 
Figura 1 - Mapa conceitual dos conceitos da TSA apresentados no presente ensaio teórico.

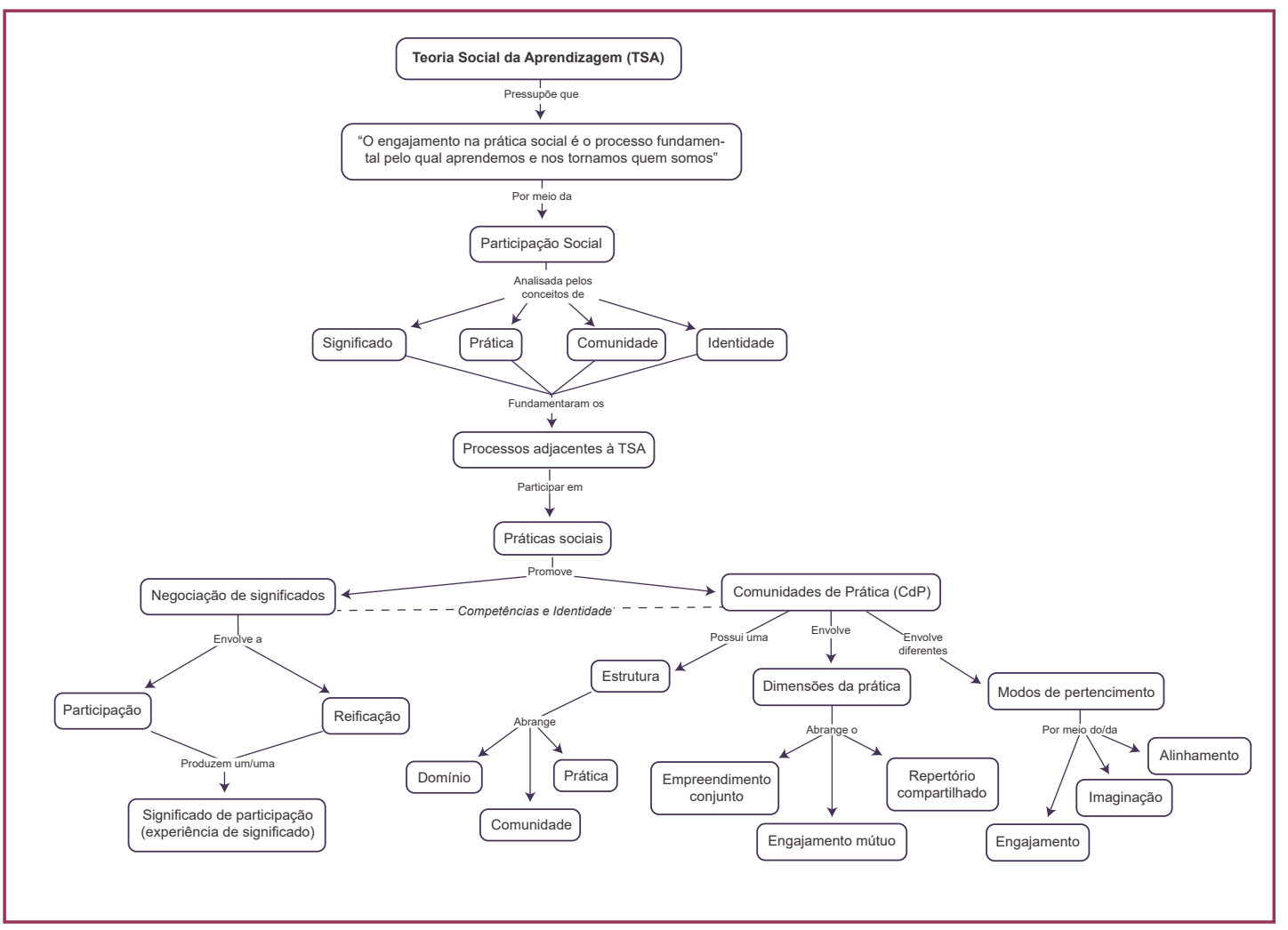

Fonte: Os autores (2020).

A Figura 1 apresenta um itinerário dos conceitos estruturantes da TSA abordados no presente ensaio teórico. Nesta perspectiva, a aprendizagem como um processo de participação social é sistematizado e analisado por meio dos conceitos de significado, prática, comunidade e identidade. Logo, a articulação destes conceitos permite uma construção teórica sobre aprendizagem que ocorre por meio da participação dos indivíduos em práticas sociais. Especificamente, são apresentados os processos de "negociação de significados" (participação e reificação) e de pertencimento à "Comunidades de Prática (CdP)" (estrutura, dimensões e modos de pertencimento), assim como suas implicações em termos de competências e identidade.

\subsection{PRESSUPOSTOS E COMPONENTES DA TSA}

O pressuposto fundamental da TSA, segundo Wenger (1998, prefácio, tradução nossa), compreende que: "O engajamento na prática social é o processo fundamental pelo qual aprendemos e nos tornamos quem somos". O foco analítico da TSA recai sobre a aprendizagem enquanto um processo de participação social, a qual se refere:

[...] não apenas ao envolvimento em determinadas atividades com certas pessoas, mas um processo mais abrangente de participação ativa nas práticas de comunidades sociais e na construção de identidades em função dessas comunidades. (WENGER, 1998, p. 4, tradução nossa).

Os grupos ou estruturas sociais que as pessoas constituem, as relações que estabelecem e as atividades que são realizadas no interior destes grupos 
definem o que o indivíduo faz (ações), quem ele é e, ainda, como ele interpreta o que faz.

Os pressupostos teóricos sobre a aprendizagem estão sintetizados em quatro princípios: a) somos seres sociais, distante de ser uma verdade trivial, este fato é um aspecto central da aprendizagem; b) o conhecimento é uma questão de competência no que diz respeito a iniciativas/empreendimentos aos quais se dá valor; c) o conhecer é uma questão de participação na busca de certos empreendimentos/ iniciativas, ou seja, de um engajamento ativo no mundo (ocorre no contexto de práticas específicas); d) o significado é a nossa habilidade de experienciar o mundo e o nosso engajamento como significativo (WENGER, 1998). Com efeito, o Significado, a Prática, a Comunidade e a Identidade correspondem aos componentes conceituais utilizados para caracterizar e analisar a aprendizagem como um processo de participação social (FIGURA 2).

Figura 2 - Componentes conceituais da TSA.

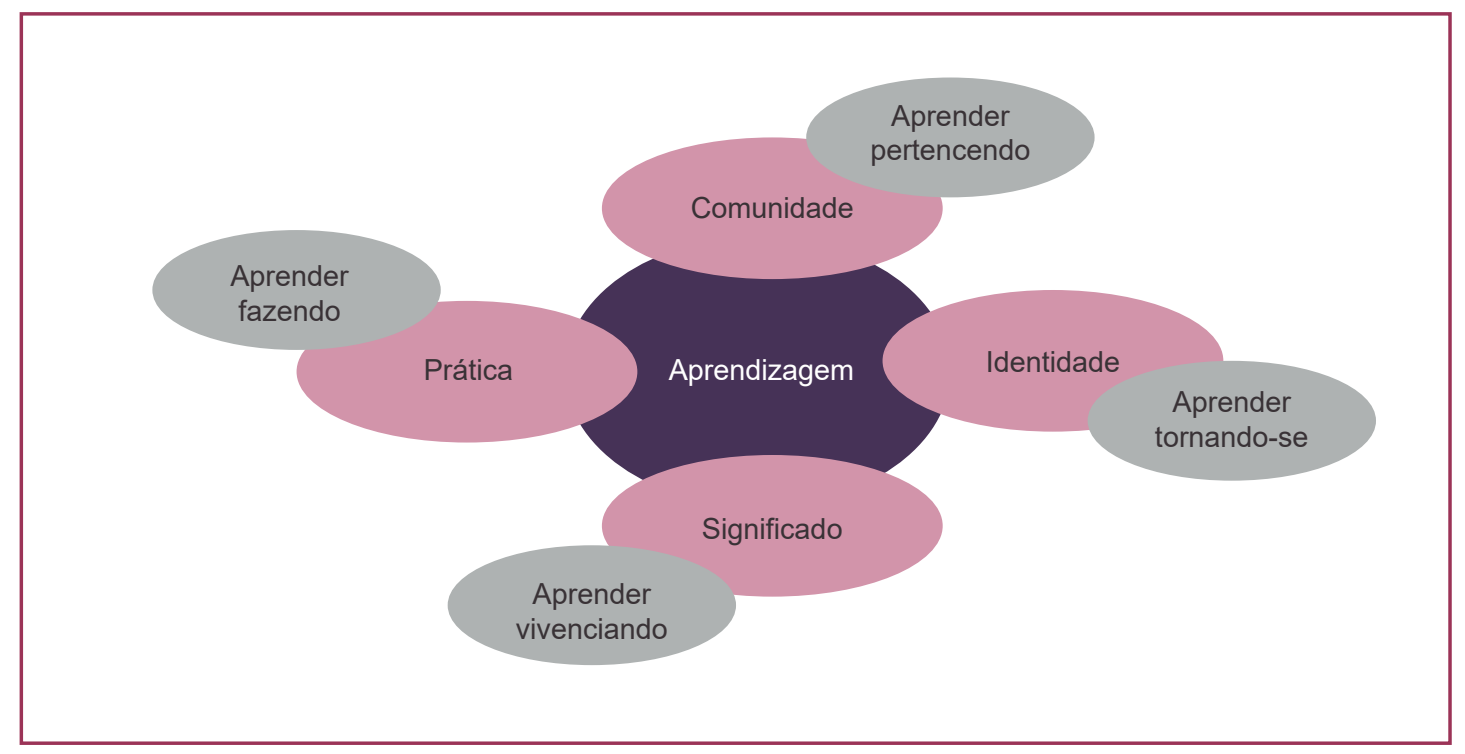

Fonte: Wenger, 1998, p. 5, tradução nossa.

O Significado diz respeito a possibilidade de analisar a capacidade de mudança individual e coletiva, para experimentar a vida e o mundo de modo significativo. A Prática corresponde ao componente que permite a análise das formas como os indivíduos compartilham os recursos históricos e sociais, assim como as perspectivas que sustentam o engajamento mútuo em uma determinada ação ou atividade. O componente Comunidade são as configurações sociais nas quais as iniciativas (projetos ou atividades) dos indivíduos são consideradas adequadas e em que a sua participação é reconhecida como competente. O conceito de Identidade, por sua vez, permite a análise dos processos pelos quais a aprendizagem modifica os indivíduos e cria trajetórias particulares de transformação, no contexto das comunidades (WENGER, 1998).

Embora a Aprendizagem tenha uma posição central no constructo teórico proposto, ao ser substituída pela posição de qualquer um dos outros componentes a figura mantém seu sentido, pois os componentes estão interligados e definem-se 
mutuamente. $O$ caráter inter-relacional desta estrutura conceitual reflete a perspectiva de uma aprendizagem que ocorre no contexto da experiência cotidiana de participação do indivíduo no mundo, em que o agente (pessoa que age), a atividade (a prática) e o ambiente, são elementos que se constituem de modo recíproco (WENGER, 1998). Esta perspectiva, portanto, permite observar a aprendizagem a partir das relações sociais do indivíduo nos diferentes contextos em que participa, assim como a aprendizagem que ocorre em um tipo particular de comunidade (Comunidade de Prática) que as pessoas constituem ao se engajarem conjuntamente em uma prática social.

\subsection{PROCESSOS ADJACENTES À TSA}

A participação do indivíduo em práticas sociais típicas promove o surgimento dos processos de negociação de significados e de formação de CdP. O conceito de prática sugere a ação "fazendo" em um contexto histórico e social que atribui estrutura e significados ao que o indivíduo faz (WENGER, 1998). Isto inclui o que é explicitado, falado e representado (linguagem, ferramentas, documentos, imagens, símbolos, papéis definidos) e também o que é implícito, subentendido e apenas assumido pelos indivíduos (relações implícitas, percepções específicas, compreensões incorporadas e visões de mundo compartilhadas). Apesar de diferentes iniciativas (atividades e ações) atribuírem distintas características às práticas sociais, elas envolvem o mesmo caráter de participação (incorporado, ativo, social, negociado e complexo) em que o agir e o conhecer são simultâneos e indissociáveis (WENGER, 2010).

\subsubsection{Negociação de significados por meio do engajamento em práticas sociais}

O enfoque atribuído ao significado na TSA o define como uma experiência da vida cotidiana, "localizado" em um processo que Wenger (1998) denomina de "negociação de significados". O termo negociação expressa o caráter de continuidade do processo em que produzimos uma nova experiência de significado àquilo que fazemos e dizemos, mesmo que estejam relacionados a algo que já fizemos e dissemos no passado. Por meio de atividades rotineiras "[...] produzimos significados que ampliam, redirecionam, rejeitam, reinterpretam, modificam ou confirmam as histórias de significado de que fazem parte" (WENGER, 1998, p. 52, tradução nossa). O processo de negociação de significados constitui-se da interação entre dois outros processos: a participação e a reificação.

O termo participação designa uma iniciativa/atividade em que o significado é estabelecido por meio de relações e identidades compartilhadas, envolvendo tomar parte das ações, estabelecer relações, o desejo de pertencer à comunidade, a compreensão mútua alcançada, o modo de partilhar histórias e recursos sociais que sustentam um engajamento mútuo na ação. A reificação consiste em converter as experiências em "coisas" (objeto material ou concreto). Wenger (1998, p. 59, tradução nossa) explica que: "As abstrações, ferramentas, símbolos, histórias, termos e conceitos que reificam ("coisificam") algo da prática em uma forma congelada/ 
materializada". Isso abrange mecanismos como "fazer, representar, nomear, codificar, perceber, interpretar, utilizar e reestruturar".

A dualidade entre estes dois processos sugere precisamente que, em termos de significado, pessoas e coisas não podem ser definidas independentes uma da outra. A participação é claramente um processo social, entretanto, compreende ainda uma experiência pessoal (individual). A reificação, por sua vez, permite que o indivíduo coordene suas ações coletivamente e também direciona a sua própria percepção sobre o mundo e sobre ele mesmo. Assim sendo, o significado da participação em práticas sociais não está no indivíduo e nem no mundo, mas na relação dinâmica e contínua de viver e tomar parte do mundo (WENGER, 1998).

2.2.2 Comunidades de Prática ( $\mathrm{CdP})$ : o tipo de comunidade que o engajamento na prática resulta

Por definição, uma CdP compreende um "[...] grupo de pessoas que compartilham um interesse em comum, um conjunto de problemas ou uma paixão por um determinado assunto/tópico, as quais aprofundam seu conhecimento e expertise nessa área, interagindo de forma contínua" (WENGER; MCDERMOTT; SNYDER, 2002, p. 4, tradução nossa). Assim, uma CdP consiste em uma estrutura social resultante do engajamento de um grupo de pessoas em práticas sociais compartilhadas, constituindo um "espaço" no qual a negociação dos significados dessas práticas entre os membros da CdP é a principal forma de aprendizagem e de constituição da identidade (WENGER, 2010).

\subsubsection{CdP: estrutura, as dimensões da prática e os modos de pertencimento}

Estruturalmente uma CdP constitui-se a partir da combinação de três componentes: o domínio, a comunidade e a prática (WENGER; MCDERMOTT; SNYDER, 2002). O domínio indica a delimitação de quais temas ou assuntos são de interesse da CdP. É o motivo pelo qual as pessoas se reúnem, com implicações no compromisso com o grupo e na definição de sua identidade e na da própria CdP. A comunidade constitui o "espaço" em que se estabelecem as interações entre os membros e que possibilita a criação de um ambiente de confiança e desafiador, favorecendo a aprendizagem. A prática diz respeito às atividades, ideias, ferramentas, informações, linguagem e histórias que os membros da CdP compartilham (WENGER-TRAYNER; WENGER-TRAINER, 2018). A coerência e a sincronia das atividades de uma CdP são sustentadas pela relação de três dimensões da prática: empreendimento conjunto, engajamento mútuo e repertório compartilhado (FIGURA 3). 
Figura 3 - Dimensões da prática como propriedade de uma CdP.

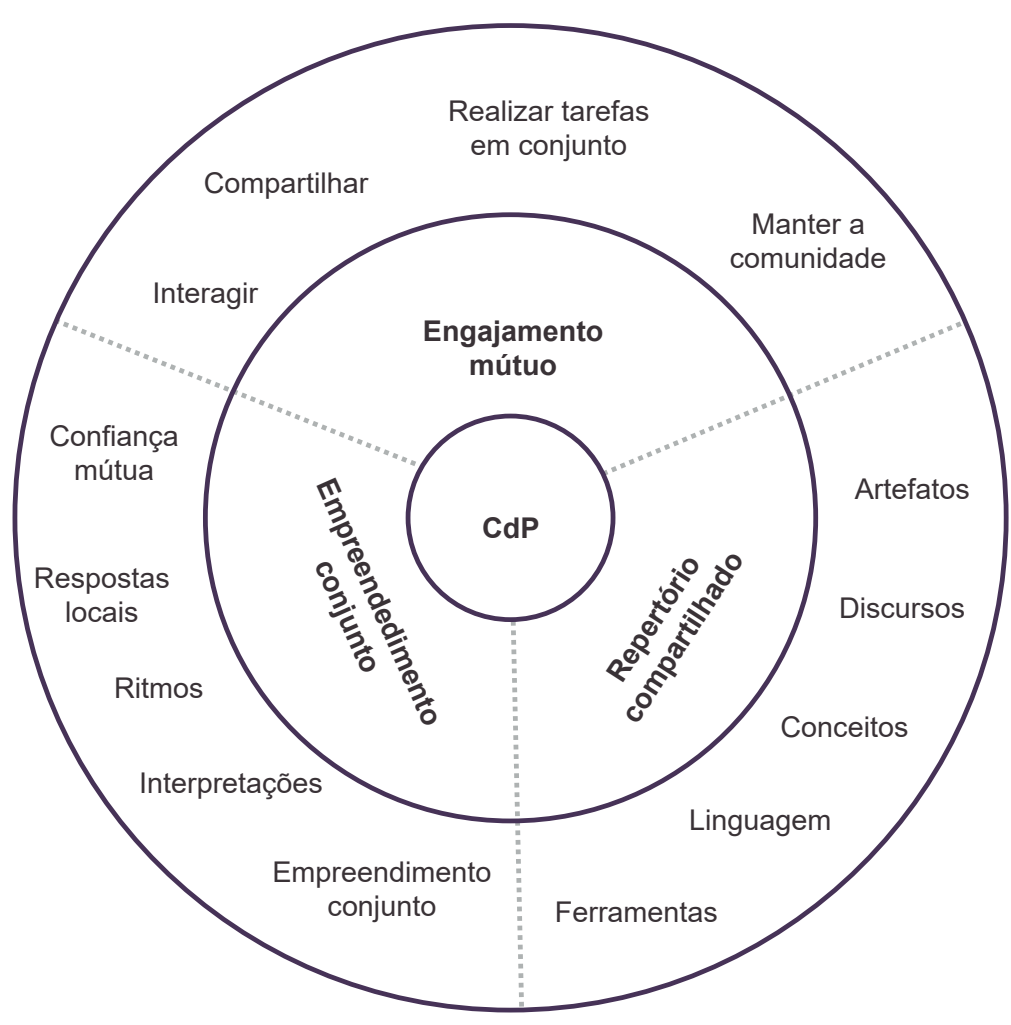

Fonte: Wenger, 1998, p. 73, tradução nossa.

O empreendimento conjunto (iniciativa ou atividade) abrange as responsabilidades que os membros da CdP assumem em conjunto pela confiança estabelecida, semelhança das interpretações realizadas e das respostas construídas localmente à CdP. O engajamento mútuo é o processo pelo qual os membros da CdP assumem vias diversas para realizar iniciativas em conjunto. A prática existe porque as pessoas se envolvem mutuamente na negociação dos significados das ações compartilhadas. Por sua vez, o repertório compartilhado diz respeito ao que se vai produzindo e consolidando coletivamente ao longo do tempo na CdP (ferramentas, rotinas, linguagem) (WENGER, 1998). O Quadro 1 apresenta os indicadores da existência de uma CdP e as dimensões da prática correspondentes.

Quadro 1 - Indicadores da existência de uma CdP.

\begin{tabular}{|c|c|}
\hline Indicadores & Dimensões da prática \\
\hline 1. Relações mútuas sustentadas - harmoniosas ou conflituosas & Engajamento mútuo \\
\hline 2. Formas compartilhadas de se envolver em fazer coisas em conjunto & $\begin{array}{c}\text { Engajamento mútuo } \\
\text { Empreendimento conjunto }\end{array}$ \\
\hline 3. Rápido fluxo de informações e de propagação da inovação & Engajamento mútuo \\
\hline $\begin{array}{c}\text { 4. Ausência de preâmbulos introdutórios, como se conversas e } \\
\text { interações fossem apenas a continuação de um processo em } \\
\text { andamento }\end{array}$ & $\begin{array}{c}\text { Engajamento mútuo } \\
\text { Repertório compartilhado }\end{array}$ \\
\hline 5. Configuração muito rápida de um problema a ser discutido & $\begin{array}{c}\text { Engajamento mútuo } \\
\text { Repertório compartilhado }\end{array}$ \\
\hline
\end{tabular}

Continua na próxima página... 
Continuação do quadro 1

\begin{tabular}{|c|c|}
\hline $\begin{array}{c}\text { 6. Coerência (hrmonia) clara nas descrições dos membros, sobre } \\
\text { quem pertence à CdP }\end{array}$ & Engajamento mútuo \\
\hline $\begin{array}{c}\text { 7. Saber o que os outros sabem, o que podem fazer e como podem } \\
\text { contribuir para um empreendimento/iniciativa }\end{array}$ & $\begin{array}{c}\text { Engajamento mútuo } \\
\text { Empreendimento conjunto } \\
\text { Repertório compartilhado }\end{array}$ \\
\hline 8. Identidades definidas mutuamente & Engajamento mútuo \\
\hline 9. Capacidade de avaliar a adequação de ações e de produtos & Repertório compartilhado \\
\hline 10. Ferramentas, representações e outros artefatos específicos & Repertório compartilhado \\
\hline $\begin{array}{c}\text { 11. Conhecimento local, histórias compartilhadas, piadas internas, } \\
\text { risos de conhecedor }\end{array}$ & Repertório compartilhado \\
\hline $\begin{array}{c}\text { 12. Jargões e atalhos para comunicação, bem como a facilidade de } \\
\text { produzir outros novos }\end{array}$ & $\begin{array}{c}\text { Repertório compartilhado } \\
\text { Engajamento mútuo }\end{array}$ \\
\hline 13. Certos estilos reconhecidos que revelam o pertencimento à CdP & Engajamento mútuo \\
\hline $\begin{array}{c}\text { 14. Discurso compartilhado refletindo uma certa perspectiva sobre o } \\
\text { mundo }\end{array}$ & Engajamento mútuo \\
\hline
\end{tabular}

Fonte: Wenger (1998, p. 125, tradução nossa)

Referente à participação do indivíduo em uma CdP, Wenger (1998) considera três distintos modos de pertencimento à $\mathrm{CdP}$ : o engajamento, a imaginação, e o alinhamento. O engajamento diz respeito ao envolvimento ativo do indivíduo no processo de negociação de significado, compartilhando histórias e interagindo com os demais membros. A imaginação compreende o processo de criar imagens do mundo e visualizar conexões que extrapolam a própria experiência de engajamento local do indivíduo. O alinhamento é um modo de pertencimento em que se coordenam as atividades compartilhadas pelos membros da CdP (empreendimento local), de modo a torná-las parte de estruturas sociais mais amplas. Portanto, a maior parte do que as pessoas fazem envolve a combinação de engajamento, imaginação e alinhamento, sendo que a ênfase em um ou outro atribui qualidades distintas às ações e aos significados destas.

\subsubsection{Os conceitos de competência e identidade na TSA}

Ao engajarem-se conjuntamente nos empreendimentos da $\mathrm{CdP}$, negociando os significados das experiências compartilhadas, os membros definem o regime de competência da CdP. Para Wenger (2010), o regime de competências compreende um conjunto de critérios e expectativas, pelo qual os membros da CdP reconhecem uns aos outros como competentes. A competência, portanto, não se refere apenas à capacidade de executar determinadas ações ou ao domínio de determinadas habilidades. Ser competente significa ser capaz de se envolver com os demais membros e utilizar de modo apropriado o reportório de recursos da CdP (WENGER, 2010). Deste modo, a perspectiva é de que a competência é definida, criada e validada no próprio processo de participação social na prática da CdP.

Similarmente, a identidade corresponde a um aspecto "[...] inseparável da prática social, da comunidade e do processo de negociação do significado, de modo que se pode falar de um em função do outro" (WENGER, 1998, p. 145, tradução nossa). Mesmo no contexto de uma prática social específica, as identidades do indivíduo não 
compreendem apenas um processo individual para essa prática, mas se definem também em virtude da posição do indivíduo como membro de $\mathrm{CdP}$ e a posição destas dentro de estruturas sociais mais amplas. As práticas, as linguagens e as visões de mundo do indivíduo refletem suas relações sociais, de modo que até mesmo em seus pensamentos mais privados ele utiliza conceitos, imagens e perspectivas que adquiriu por meio da sua participação em diferentes CdP (WENGER, 2010).

Considerar a identidade em sua face social implica reconhecer que as pessoas são o reflexo da participação contínua em CdP às quais pertenceram e/ou pertencem (WENGER, 2010). A identidade vai além de uma única trajetória temporal de participação, ela abrange a integração (nexo) das filiações (participações) a diferentes CdP, assim como os diversos modos de pertencimento (engajamento, imaginação e alinhamento) do indivíduo nestas CdP. Para Wenger (1998), múltiplas trajetórias em diferentes CdP se tornam parte uma da outra por meio de conexões ou da reconciliação das experiências e das formas de afiliação do indivíduo, transformando (resignificando) quem ele é e suas histórias.

\subsection{EVOLUÇÃO DA TSA E DO CONCEITO DE CdP}

Com base na proposta inicial de Wenger (1998) sobre a teorização do conceito de CdP para a análise da aprendizagem, Wenger, Mcdermott e Snyder (2002) atribuíram ao conceito de CdP uma perspectiva instrumental que fornece um quadro conceitual para guiar o desenvolvimento de CdP. Tal iniciativa buscou atender a demanda de fomentar a inovação de práticas profissionais e a solução criativa de problemas típicos do contexto de intervenção. A este processo foram introduzidos os papéis de líderes e/ou facilitadores no desenvolvimento de CdPs.

A constatação de que uma CdP integra sistemas sociais mais amplos e complexos (social learning systems), envolvendo outras CdPs e também outras estruturas sociais (equipes, projetos e instituições) (WENGER, 2010), repercutiu na expansão do escopo conceitual da TSA em dois direcionamentos. Primeiro, no desenvolvimento da metáfora de "paisagem de prática" (landscape of practice), a qual propõem um aparato conceitual para analisar a multifiliação em diferentes CdPs e os processos emergentes da trajetória de participação do indivíduo em múltiplas estruturas sociais (WENGER-TRAYNER et al., 2015). Segundo, na delimitação de um conjunto de outros tipos de estruturas sociais (discussões, workshops, networks, equipes e CdPs), além de CdP, pelos quais as pessoas aprendem de modo intencional, denominado de "espaço de aprendizagem social" ${ }^{3}$ (social learning space) (WENGERTRAYNER; WENGER-TRAYNER, 2018).

Em fase recente, Wenger-Trayner et al. (2019) apresentam versão revisada do value creation framework, proposto anteriormente por Wenger, Trayner e De Laat (2011), que consiste em uma estrutura conceitual para promover e avaliar o valor que a CdP e os networks podem produzir em seus membros. O value creation framework

\footnotetext{
3 "Um espaço de aprendizagem social é similar a uma CdP, mas sem as expectativas de continuidade e compromisso contínuo. Alguns exemplos são: um grupo de pessoas que compartilha a necessidade de explorar um problema e se reunir para falar sobre o assunto; uma pessoa identificou um problema que possui em comum com outras pessoas e propôs videoconferências para encontrar uma solução." (WENGER-TRAYNER; WENGER-TRAYNER, 2018, p. 43, tradução nossa).
} 
sugere a triangulação de diferentes tipos (quantitativo e qualitativo) e fontes de dados (registros de acesso a sites, de download de documentos e histórias contadas pelos participantes), para a obtenção de evidências empíricas sobre como e quais valores as CdPs e os networks podem gerar para cada um de seus membros, bem como para as organizações/entidades (WENGER-TRAYNER; WENGER-TRAYNER, 2018).

\section{A TSA E CdP NAS PESQUISAS COM FOCO NA APRENDIZAGEM DE TREINADORES ESPORTIVOS}

O reconhecido potencial da TSA e do conceito de CdP têm contribuído nas investigações científicas com foco no desenvolvimento profissional em áreas como ciência da informação, administração, saúde e educação (LI et al., 2009). As pesquisas realizadas nessas áreas têm impulsionado estudos no âmbito do treinador esportivo, sobretudo, por considerar o caráter informal e social da aprendizagem e por reconhecer a relevância das interações sociais na construção dos conhecimentos para a intervenção profissional e na constituição da identidade do treinador (CULVER; TRUDEL, 2008).

Pesquisas com foco na trajetória de vida de treinadores de ginástica artística (BRASIL et al., 2018) e de surfe (BRASIL, 2019) revelam que suas experiências se constituíram a partir das relações sociais e das práticas em que eles se engajaram, em diferentes CdPs (família, clube, escola, atletas etc.). O regime de competências e os significados desenvolvidos em cada CdP impulsionou o engajamento em práticas sociais de outras $\mathrm{CdPs}$, atribuindo renovados significados a elas e constituindo uma trajetória de identidade. Embora se reconheça a limitação dos estudos retrospectivos para a obtenção de detalhes das experiências dos treinadores, a TSA e o conceito de CdP são ferramentas úteis para analisar o processo de ser e se tornar treinador, com possibilidades de compreensão sobre o processo de constituição das crenças, das competências e da identidade dos treinadores (CASSIDY; ROSSI, 2006).

No âmbito dos sistemas esportivos, verifica-se a existência de CdPs de atletas, treinadores e dirigentes (CULVER; TRUDEL, 2008). Ao investigarem as interações entre atletas e treinadores de uma mesma equipe de hóquei no gelo, a partir do conceito de social learning system, Galipeau e Trudel (2006) constataram a existência de duas CdPs distintas (atletas e treinadores). A delimitação dos empreendimentos de cada $\mathrm{CdP}$, assim como as relações baseadas na transparência e na "negociabilidade" entre treinadores e atletas, preservou os interesses de ambas as CdPs, fazendo com que cada uma auxiliasse no aprimoramento da prática da outra. Em contrapartida, Lemyre, Trudel e Durand-Bush (2007) verificaram a ausência de CdP entre treinadores de uma mesma liga esportiva, pois eles não interagiam regularmente para compartilhar experiências e discutir problemas comuns de treinamento, de modo que estas iniciativas eram restritas às suas equipes e clubes.

Mais recentemente, Duarte, Culver e Paquette (2020) utilizaram o conceito de "paisagem de prática" (landscape of practice) para analisar os recursos e as barreiras de aprendizagem de treinadores de curling em cadeiras de rodas filiados à federação canadense desta modalidade. Os resultados evidenciaram que o 
isolamento geográfico e os altos custos dos cursos para os treinadores foram os principais obstáculos para a aprendizagem. A noção de paisagem de prática permitiu uma visão ampla do sistema esportivo em que os treinadores estavam vinculados, indicando estratégias nos diferentes níveis deste sistema para minimizar as barreiras identificadas, nomeadamente de estabelecer interações com pessoas influentes definidas a critério dos próprios treinadores e de utilizar as oportunidades em "acampamentos" de treinamento e competições (camps) para fomentar as interações pessoais.

De fato, as pesquisas de caráter analítico apresentam potencial para a identificação da existência ou não de CdPs, assim como para explorar a "paisagem de prática" de treinadores em diferentes contextos esportivos (equipes, competições, entidades e modalidades). As contribuições desses estudos abrangem a obtenção de evidências sobre quais aspectos contribuem e/ou dificultam o engajamento mútuo dos treinadores em empreendimentos compartilhados, assim como fornecem informações preliminares para estudos experimentais que buscam projetar, implementar e sustentar CdPs de treinadores (DUARTE; CULVER; PAQUETTE, 2020).

As investigações com foco no desenvolvimento de CdPs têm sido, destacadamente, recorrentes na agenda investigativa sobre o treinador. CdPs de treinadores foram desenvolvidas no beisebol (CULVER; TRUDEL; WERTHNER, 2009; KUKLICK et al., 2016), esqui alpino (CULVER; TRUDEL, 2006; GARNER; HILL, 2017), patinação artística (CALLARY, 2013), futebol (GOMES, 2015) e com treinadores do esporte universitário (BERTRAM; GILBERT, 2011). Estes estudos revelam que as interações locais "um-a-um" favorecem a negociação de significados relacionados às demandas de intervenção e ao interesse dos treinadores em aprimorarem determinados conhecimentos. Particularmente, as reflexões em grupo levaram os treinadores ao desenvolvimento da inteligência emocional e a implementarem abordagens de intervenção centradas no atleta (GARNER; HILL, 2017; KUKLICK et al., 2016).

Integrar uma mesma entidade ou clube esportivo não garante a formação de CdP entre treinadores (CULVER; TRUDEL, 2006), visto que a cultura competitiva do esporte (CULVER; TRUDEL; WERTHNER, 2009) e o desequilíbrio de poder nas relações sociais neste contexto (GOMES et al., 2013) podem impedir o engajamento mútuo para o aprimoramento de suas perspectivas de intervenção profissional. Algumas recomendações para o desenvolvimento de CdPs de treinadores abrangem: ter a presença de um "facilitador" reconhecido pela entidade esportiva, familiarizado com o domínio da CdP e capaz de "nutri-la" (GILBERT; GALLIMORE; TRUDEL, 2009); considerar os treinadores, os dirigentes e o facilitador para a estruturação da CdP (BARNSON, 2010); incentivar a colaboração e a comunicação contínuas; promover um ambiente em que os treinadores se sintam confiantes e abertos ao aprendizado (CALLARY, 2013); e ainda, a criação de CdPs locais (equipes e clubes) (KUKLICK et al., 2016).

Alguns estudos realizados em contexto universitário revelam que a presença de um facilitador para desenvolver e "nutrir" a CdP se configura como requisito para tal iniciativa. Ao desenvolver uma CdP de graduandos estagiários, Gomes (2015) 
verificou que a sua intervenção como pesquisador/facilitador, propondo estratégias para minimizar a assimetria da participação dos estagiários, potencializou o engajamento deles nas iniciativas da CdP e, consequentemente, na negociação de significados e reflexões críticas sobre o domínio de interesse da CdP. Por outro lado, Stoszkowski e Collins (2014), ao utilizarem um blog como recurso para desenvolver uma CdP de treinadores em formação, constataram que a falta de autonomia de alguns deles para o processo reflexivo limitou o engajamento mútuo nas inciativas/ empreendimentos do grupo, dificultando a negociação de significados e o surgimento da CdP entre os acadêmicos.

Um dos principais desafios dos estudos que buscam desenvolver CdPs têm sido mediar o engajamento mútuo dos treinadores em iniciativas compartilhadas na CdP. Embora seja evidente que as configurações dos sistemas esportivos reforçam a ideia de treinadores como rivais, ressalva-se que a aprendizagem por meio da participação em CdPs não diminui a relevância da competição e do êxito esportivo (GILBERT; GALLIMORE; TRUDEL; 2009). Ademais, fomentar a participação dos treinadores em outros tipos de espaços de aprendizagem social (networks, discussões, workshops, clínicas) pode levá-los à constituição de futuras CdPs. No contexto universitário, destaca-se que os diferentes interesses entre os membros da $\mathrm{CdP}$ e o caráter obrigatório dos temas propostos podem dificultar que os treinadores se engajem na negociação de significados a ponto de modificarem seus conhecimentos para a atuação profissional (JONES; MORGAN; HARRIS, 2012).

$\mathrm{O}$ argumento de que a aprendizagem em CdP implica exclusivamente resultados coletivos tem sido uma advertência recorrente na literatura especializada (MALLETT, 2010). Por outro lado, pesquisas que utilizaram o value creation framework evidenciam a criação de valores imediatos pessoais, desenvolvidos por meio da participação dos treinadores em CdP, ligados às suas competências para a intervenção pedagógica (BERTRAM; CULVER; GILBERT, 2016; 2017). Em estudo com treinadores canadenses de curling em cadeiras de rodas, Duarte (2020) destacou que todos os membros da CdP obtiveram valores pessoais ligados ao senso de inclusão, obtenção de outros pontos de vista e interações com treinadores de outras gerações (novatos e experientes). Os resultados de tais estudos sugerem que abordagens longitudinais possam contribuir para a verificação de valores de aprendizagem mais complexos e demorados de serem desenvolvidos.

\subsection{PERSPECTIVAS E DESAFIOS À PESQUISA CIENTÍFICA}

O potencial da TSA para a análise e promoção da aprendizagem do treinador tem aumentado nos últimos anos, sobretudo por considerar que o engajamento em práticas sociais é o processo central pelo qual a aprendizagem ocorre (CASSIDY; ROSSI, 2006). A perspectiva é de que o "corpo de conhecimento" para a intervenção profissional do treinador corresponde a uma estrutura "viva", constituída por um complexo sistema de inter-relação entre CdPs e demais espaços de aprendizagem social dos quais os treinadores participam. Deste modo, as suas competências e a sua identidade resultam da sua "viagem" particular nesta complexa paisagem (WENGER-TRAYNER et al., 2015). Esta perspectiva atende ao caráter contextual e 
social da intervenção pedagógica do treinador, apresentando aptidão para analisar a natureza pragmática e problemática da sua aprendizagem (CULVER; KRAFT, 2017).

A amplitude da aproximação que a TSA propõe ao fenômeno da aprendizagem fundamenta-se na integração de diversas teorias que possuem aspectos comuns (WENGER, 2010). Contudo, Wenger-Trayner (2013) adverte sobre a necessidade de se compreender os pressupostos de cada teoria, o seu foco de análise, a sua linguagem técnica e também com que tipo de argumentação teórica e científica busca contribuir. Referente ao processo investigativo, esta abordagem permite ao investigador integrar componentes conceituais que sejam úteis para "contar histórias" mais completas ou novas sobre a aprendizagem (WENGER-TRAYNER, 2013), com implicações na sua posição epistemológica e nas suas decisões metodológicas (CULVER; KRAFT, 2017).

Uma possibilidade recorrente tem sido a adoção de paradigmas de pesquisa participativa e pós-positivista, permitindo diferentes níveis de análise e de abordagem metodológica (quantitativa, qualitativa e mista) (WENGER-TRAYNER et al., 2019). Com particular atenção às pesquisas de método misto, a ressalva é para as inconsistências que resultam da integração entre os componentes do processo investigativo de pesquisas quantitativas e qualitativas. Isso não significa que contradições não devam ser aceitas ou que devam ser consideradas uma fragilidade típica de pesquisas desta abordagem (DENSCOMBE, 2008). A recomendação, portanto, é de que os tipos de instrumentos e procedimentos de pesquisa devem ser selecionados e justificados a partir de pressupostos epistemológicos claros e também do seu "valor prático" para lidar com o problema específico de estudo (WENGERTRAYNER et al., 2019).

Outras recomendações metodológicas específicas abrangem explorar e documentar, detalhadamente, como as CdPs são planejadas e implementadas em ambientes reais ( $\mathrm{LI}$ et al., 2009). O pesquisador enquanto facilitador no desenvolvimento de CdPs de treinadores, mediante uma abordagem não prescritiva, pode promover um ambiente seguro para a aprendizagem e potencializar a negociação de significados entre os treinadores (CULVER; TRUDEL; WERTHNER, 2009). Uma visão sistêmica do contexto de intervenção destes profissionais (social learning systems e landscape of practice) pode permitir ao facilitador envolver nas iniciativas da CdP os diferentes níveis do sistema esportivo para melhor atender as necessidades de aprendizagem dos treinadores (DUARTE; CULVER; PAQUETTE, 2020). Sugere-se ainda períodos mais longos para coleta de dados, abrangendo uma temporada esportiva completa, para se obter uma compreensão mais detalhada de como o conhecimento é desenvolvido pelos treinadores ao participarem da CdP (GARNER; HILL, 2017; STOSZKOWSKI; COLLINS, 2014).

As tecnologias de informação e de comunicação compreendem instrumentos que auxiliam na promoção de CdPs e outros espaços de aprendizagem social, assim como para a obtenção de dados (quantitativos e qualitativos) referentes a aprendizagem dos treinadores. As plataformas da web 2.0 (blogs, salas de bate-papo e redes sociais) permitem que os treinadores participem em ambientes virtuais de aprendizagem social e recebam suporte constante para seu desenvolvimento, com níveis mais baixos 
de custo financeiro, menos tempo e entre longas distâncias (DUARTE; CULVER; PAQUETTE, 2020; STOSZKOWSKI; COLLINS, 2014). O desafio é implementar recursos tecnológicos variados de interação social entre os treinadores que atendam as potencialidades e as especificidades de cada contexto de aprendizagem.

As principais demandas investigativas são analisar como o tempo influencia no estabelecimento de uma $\mathrm{CdP}$ e no processo de desenvolvimento da identidade dos treinadores dentro de cursos/programas online; e também como estimular o engajamento mútuo dos treinadores em iniciativas compartilhadas por meio de recursos tecnológicos; (STOSZKOWSKI; COLLINS, 2014). Embora um amplo gradiente de recursos tecnológicos indique novas ferramentas para a interação social, elas não compreendem a própria CdP ou o espaço de aprendizagem social (WENGER, 2010). Além do mais, quando implementadas pelo pesquisador/facilitador, devem ser consideradas as rotinas e o ritmo natural de desenvolvimento da CdP ou de outras formas de organização social (ROCHA; PEREIRA, 2017).

A utilização da TSA nos estudos com foco na aprendizagem do treinador esportivo traduz uma ampliação dos direcionamentos epistemológicos, conceituais e investigativos nessa área. Considerar a aprendizagem como um processo de participação em práticas sociais compreende uma importante alternativa para superar a perspectiva da aprendizagem fundamentada, exclusivamente, na aquisição direta e reprodução descontextualizada de conhecimentos e competências. A TSA fornece uma matriz analítica e instrumental para se compreender e promover a aprendizagem de treinadores, em uma proposta que atende ao dinamismo dos contextos de intervenção pedagógica no âmbito esportivo e às demandas atuais de comunicação e interação social.

\section{CONSIDERAÇÕES FINAIS}

A perspectiva apresentada neste ensaio teórico integra um cenário atual de debate científico acerca da aprendizagem e desenvolvimento profissional do treinador esportivo. Ressaltou-se que a TSA oferece um amplo escopo analítico e instrumental, configurando-se como um continuum, que em um extremo permite a análise e a promoção da aprendizagem enquanto participação em uma CdP; expandindo-se para o outro extremo, no qual a aprendizagem ocorre (ou pode ser promovida), a partir das diferentes práticas e relações sociais que o treinador se engaja, em função de uma "paisagem de prática" vasta e complexa.

Os direcionamentos temáticos emergentes na agenda investigativa sobre o treinador esportivo refletem a amplitude do quadro conceitual que constitui a TSA. As contribuições destes estudos contemplam a demanda de se utilizar matrizes teóricas que preservem a complexidade, a natureza social e o caráter contextual da aprendizagem dos treinadores. Estudos com desenhos metodológicos mistos acenam a possibilidade de maior abrangência dos dados obtidos, porém de elevada complexidade aos pesquisadores. Há em curso um processo interativo e dinâmico entre a obtenção de dados empíricos sobre a aprendizagem e o aprimoramento constante da TSA. 
Embora se considere que os temas foram abordados de modo suficiente, sem pretensão de ser uma análise conclusiva nas discussões efetuadas, uma série de possíveis temas para apresentação e para uma discussão mais aprofundada relacionada a TSA podem emergir do conteúdo disponibilizado neste ensaio teórico. Além disso, o itinerário proposto compreende um entendimento presente, passível de renovadas argumentações e interpretações.

\section{REFERÊNCIAS}

ARMOUR, Kathleen. The learning coach...the learning approach: professional development for sports coach professionals. In: LYLE, John; CUSHION, Christopher (org.). Sports coaching: professionalism and practice. Edinburgh: Churchill Livingstone, 2010. p. 153-164.

BARNSON, Steven. Communities of coaches: the missing link. Journal of Physical Education, Recreation and Dance, v. 81, n. 7, p. 25-37, 2010.

BERTRAM, Rachael; GILBERT, Wade. Learning communities as continuing professional development for sport coaches. Journal of Coaching Education, v. 4, n. 2, p. 40-61, 2011.

BERTRAM, Rachael; CULVER, Diane M.; GILBERT, Wade. Creating value in a sport coach community of practice: a collaborative inquiry. International Sport Coaching Journal, v. 3, n. 1, p. 2-16, 2016.

BERTRAM, Rachael; CULVER, Diane M.; GILBERT, Wade. A university sport coach community of practice: using a value creation framework to explore learning and social interactions. International Journal of Sports Science and Coaching, v. 12, n. 3, p. 287302, 2017.

BRASIL, Vinicius Zeilmann. A construção do conhecimento como um processo de participação social: estudo de caso com uma treinadora de surf. 2019. $411 \mathrm{f}$. Tese (Doutorado em Educação Física) - Centro de Desportos, Universidade Federal de Santa Catarina, Florianópolis, 2019.

BRASIL, Vinicius Zeilmann et al. A trajetória de vida de treinadores de ginástica artística. Journal of Physical Education, v. 29, n. 1, e-2933, 2018. Disponível em: https:// periodicos.uem.br/ojs/index.php/RevEducFis/article/view/35557. Acesso em: 18 maio 2021.

BRASIL, Vinicius Zeilmann et al. A trajetória de vida do treinador esportivo: as situações de aprendizagem em contexto informal. Movimento, v. 21, n. 3, p. 815-829, 2015.

CALLARY, Bettina. Coaches create and sustain a community of practice within a club. Phenex Journal, v. 4, n. 3, p. 1-13, 2013. Disponível em: http://ojs.acadiau.ca/index. php/phenex/article/download/1497/1260. Acesso em: 18 maio 2021.

CALLARY, Bettina; WERTHNER, Penny; TRUDEL, Pierre. Exploring coaching actions on developed values: a case study of a female hockey coach. Journal of Lifelong Education, v. 32, n. 2, p. 209-229, 2013.

CASSIDY, Tania; ROSSI, Tony. Situated learning: (re)examining the notion of apprenticeship in coach education. International Journal of Sports Science and Coaching, v. 1, n. 3, p. 235-246, 2006. 
CULVER, Diane; KRAFT, Erin. Beyond 'Crude Pragmatism' in sport coaching: insights from CS Peirce, William James, and John Dewey: a commentary. International Journal of Sports Science and Coaching, v. 12, n. 1, p. 40-42, 2017.

CULVER, Diane; TRUDEL, Pierre. Clarifying the concept of Communities of Practice in Sport. International Journal of Sports Science and Coaching, v. 3, n. 1, p. 1-10, 2008.

CULVER, Diane; TRUDEL, Pierre. 'Cultivating coaches' communities of practice. In: JONES, Robyn (org.). The sports coach as educator: re-conceptualizing sports coaching. London: Routledge, 2006. p. 97-112.

CULVER, Diane; TRUDEL, Pierre; WERTHNER, Penny. A sport leader's attempt to foster a coaches' community of practice. International Journal of Sports Science and Coaching, v. 4, n. 3, p 365-383, 2009.

CUSHION, Christopher; TOWNSEND, Robert. Jean Lave: learning in coaching as social praxis. In: NELSON, Lee; GROOM, Ryan; POTRAC, Paul (orgs.). Learning in sports coaching: theory and application. Abingdon: Routledge, 2016. p. 139-148.

CUSHION, Christopher; TOWNSEND, Robert. Technology-enhanced learning in coaching: a review of literature. Educational Review, v. 71, n. 5, p. 631-649, 2019.

DENSCOMBE, Martyn. Communities of practice: A research paradigm for the mixed methods approach. Journal of mixed methods research, v. 2, n. 3, p. 270-283, 2008.

DUARTE, Tiago. A Collaborative effort to frame and assess a social learning space for wheelchair curling coaches. 2020. $281 \mathrm{f}$. Thesis (Doctor of Philosophy) - Faculty of Health Sciences, University of Ottawa, Ottawa, 2020.

DUARTE, Tiago; CULVER, Diane M.; PAQUETTE, Kyle. Mapping Canadian wheelchair curling coaches' development: a landscape metaphor for a systems Approach. International Sport Coaching Journal, v. 1, n. 2, p. 1-10, 2020.

GALIPEAU, James; TRUDEL, Pierre. Athlete learning in a community of practice: is there a role for the coach? In: JONES, Robyn (org.). The sports coach as educator: reconceptualizing sports coaching. London: Routledge, 2006. p. 77-94.

GARNER, Paul; HILL, Denise M. Cultivating a community of practice to enable coach development in alpine ski coaches. International Sport Coaching Journal, v. 4, n. 1, p. 6375, 2017.

GILBERT, Wade. GALLIMORE, Ronald; TRUDEL, Pierre. A learning community approach to coach development in youth sport. Journal of Coaching Education, v. 2, n. 2, p. 1-21, 2009.

GILBERT, Wade; TRUDEL, Pierre. Analysis of coaching science research published from 1970-2001. Research Quarterly for Exercise and Sport. v. 75, p. 388-399, 2004.

GOMES, Rúben Emanuel Correia. Formação de treinadores no contexto académico: aprendizagem em comunidade de prática no decurso do estágio. 2015, 245 f. Tese (Doutorado em Ciência do Desporto) - Faculdade de Desporto, Universidade do Porto, Porto, 2015.

GOMES, Rúben Emanuel Correia et al. Establishing 'communities of practice': a benign or power-ridden process?'. Revista Mineira de Educação Física, n. 9, p. 886-892, 2013. Edição Especial. 
JONES, Robyn; MORGAN, Kevin; HARRIS, Kerry. Developing coaching pedagogy: seeking a better integration of theory and practice. Sport, Education and Society, v. 17, n. 3, p. 313-329, 2012.

KUKLICK, Clayton et al. A case study of one high performance baseball coach's experiences within a learning community. Qualitative research in sport, exercise and health, v. 8, n. 1, p. $61-78,2016$.

LAVE, Jean; WENGER, Etienne. Situated learning: legitimate peripheral participation. Cambridge: Cambridge University, 1991.

LEMYRE, François; TRUDEL, Pierre; DURAND-BUSH, Natalie. How youth-sport coaches learn to coach. The Sport Psychologist, n. 2, p.191-209, 2007.

LI, Linda et al. Evolution of Wenger's concept of community of practice. Implementation science, v. 4, n. 1, p. 11, 2009.

MALLETT, Clifford. Becoming a high-performance coach: pathways and communities. In: LYLE, John; CUSHION, Christopher (orgs.). Sports coaching: Professionalism and practice. Edinburgh: Churchill Livingstone, 2010. p. 119-134.

MALLET, Clifford et al. Formal vs. Informal Coach Education. International Journal of Sports Science and Coaching, v. 4, n. 3, p. 325-364, 2009.

PALINCSAR, Sullivan. Social constructivist perspectives on teaching and learning. Annual Review of Psychology, v. 49, n. 1, p. 345-375, 1998.

ROCHA, Antonieta; PEREIRA, Alda. Matriz metodológica para análise de comunidades virtuais de prática. Revista Lusófona de Educação, n. 36, p. 81-97, 2017.

STOSZKOWSKI, John; COLLINS, Dave. Communities of practice, social learning and networks: exploiting the social side of coach development. Sport, Education and Society, v. 19, n. 6, p. $773-788,2014$.

TRUDEL, Pierre; GILBERT, Wade. Coaching and coach education. In: KIRK, David, O'SULLIVAN, Mary; McDONALD, Doune (orgs.). Handbook of Physical Education. Sage: London, 2006. p. 516-539.

WALKER, Lauren; THOMAS, Rebecca; DRISKA, Andrew. Informal and nonformal learning for sport coaches: a systematic review. International Journal of Sports Science and Coaching, v. 13, n. 5, p. 694-707, 2018.

WENGER, Etienne. Communities of practice: learning, meaning, and identity, Cambridge: Cambridge University, 1998.

WENGER, Etienne. Conceptual tools for CoPs as Social Learning Systems: Boundaries, identity, trajectories and participation. In: BLACKMORE, Chris (org.). Social learning systems and communities of practice. London: Springer, 2010. p. 125-144.

WENGER, Etienne; McDERMOTT, Richard; SNYDER, William. Cultivating communities of practice: a guide to managing knowledge. Boston: Harvard Business School, 2002.

WENGER, Etienne; TRAYNER, Beverly; DE LAAT, Maarten. Promoting and assessing value creation in communities and networks: a conceptual framework. Rapport 18, Ruud de Moor Centrum, The Netherlands: Open University, 2011.

WENGER-TRAYNER, Beverly et al. Boundaries and boundary objects: an evaluation framework for mixed methods research. Journal of Mixed Methods Research, v. 13, n. 3 , p. 321-338, 2019. 
WENGER-TRAYNER, Etienne. The practice of theory: confessions of a social learning theorist. In: FARNSWORTH, V; SOLOMON, Y. (ed.). Reframing educational research: Resisting the "what works" agenda. London: Routledge, 2013. p. 105-118.

WENGER-TRAYNER, Etienne et al. Learning in landscapes of practice: boundaries, identity, and practice-based learning. London: Routledge, 2015.

WENGER-TRAYNER, Etienne; WENGER-TRAYNER, Beverly. Communities of practice handbook: three frameworks for managing an initiative, cultivating a community, and assessing the values. Version 5.0, 2018. 
Abstract: This theoretical essay presents a set of concepts from the Social Theory of Learning (STL) and discusses its implications for research focused on sports coaches' learning and development. The STL provides a broad analytical and instrumental scope with potential for understanding and promoting coaches' learning. The main thematic focuses of the research agenda in this field reflect the STL's conceptual framework. The contributions made by these studies meet the demand for using theoretical frameworks that preserve coaches learning' complexity, social nature and contextual issues.

Keywords: Learning. Physical Education and Training. Sports. Social theory.

Resumen: El objetivo de este ensayo teórico fue presentar la Teoría Social del Aprendizaje (TSA) y mostrar cómo el cuadro conceptual adyacente ha sido utilizado en las investigaciones sobre el aprendizaje y el desarrollo de entrenadores deportivos. La TSA ofrece un marco analítico e instrumental amplio, con potencial para la comprensión y promoción del aprendizaje del entrenador. Los rumbos temáticos que emergen en la agenda investigativa del área reflejan la amplitud del marco conceptual que constituye la TSA. Los aportes de estos estudios contemplan la demanda de utilizar matrices teóricas que preserven la complejidad, la naturaleza social y el carácter contextual del aprendizaje de los entrenadores.

Palabras clave: Aprendizaje. Educación Física y entrenamiento. Deportes. Teoría social. 


\section{LICENÇA DE USO}

Este é um artigo publicado em acesso aberto (Open Access) sob a licença Creative Commons Atribuição 4.0 Internacional (CC BY 4.0), que permite uso, distribuição e reprodução em qualquer meio, desde que o trabalho original seja corretamente citado. Mais informações em: http://creativecommons.org/licenses/by/4.0

\section{CONFLITO DE INTERESSES}

Os autores declararam que não existe nenhum conflito de interesses neste trabalho.

\section{CONTRIBUIÇÕES AUTORAIS}

Vinicius Zeilmann Brasil: Escrita do texto, estudo da teoria, seleção e análise dos estudos sobre a aprendizagem do treinador esportivo.

Valmor Ramos: Análise e revisão geral do texto.

Ana Flávia Backes: Escrita, formatação e revisão geral do texto.

Leonardo Ristow: Escrita, formatação e revisão geral do texto.

Michel Milistetd: Estudo da teoria, análise das pesquisas sobre a aprendizagem do treinador esportivo e revisão geral do texto.

Juarez Vieira do Nascimento: Análise e revisão geral do texto.

\section{FINANCIAMENTO}

O presente trabalho foi realizado com apoio da Coordenação de Aperfeiçoamento de Pessoal de Nível Superior - Brasil (CAPES) - Código de Financiamento 001. "This study was financed in part by the Coordenação de Aperfeiçoamento de Pessoal de Nível Superior - Brasil (CAPES) - Finance Code 001"

\section{COMO REFERENCIAR}

BRASIL, Vinicius Zeilmann; RAMOS,Valmor; BACKES, Ana Flávia; RISTOW, Leonardo; MILISTETD, Michel; NASCIMENTO, Juarez Vieira do. Aprendizagem do treinador esportivo como um processo de participação social: uma perspectiva à pesquisa científica. Movimento (Porto Alegre), v.27, p.e27027, jan./dez. 2021. Disponível em: https://seer.ufrgs.br/Movimento/article/view/105388. Acesso em: [dia] [mês abreviado]. [ano]. DOI: https://doi.org/10.22456/1982-8918.105388

\section{RESPONSABILIDADE EDITORIAL}

Alex Branco Fraga*, Elisandro Schultz Wittizorecki*, Ivone Job*, Mauro Myskiw*, Raquel da Silveira*

*Universidade Federal do Rio Grande do Sul, Escola de Educação Física, Fisioterapia e Dança, Porto Alegre, RS, Brasil. 